\title{
PREVALÊNCIA AMBULATORIAL EM UM HOSPITAL GERAL DE MARCADORES PARA HEPATITES B E C EM PACIENTES COM INFECÇÃO PELO VÍRUS DA IMUNODEFICIÊNCIA HUMANA
}

\author{
Cristiane Valle TOVO ${ }^{1}$, Diogo Edele dos SANTOS ${ }^{2}$, Ângelo Zambam de MATTOS ${ }^{2}$, \\ Paulo Roberto Lerias de ALMEIDA ${ }^{1}$, Angelo Alves de MATTOS $^{2}$ e Breno Riegel SANTOS ${ }^{1}$
}

RESUMO - Racional - Os vírus das hepatites B (VHB) e C (VHC) e da imunodeficiência humana (HIV) utilizam a mesma rota de transmissão, sendo a prevalência de VHB e VHC em pacientes infectados pelo HIV maior do que aquela encontrada na população em geral. Objetivo - Determinar a prevalência de marcadores para hepatites B e C em uma população de pacientes com HIV, bem como os fatores de risco envolvidos. Pacientes e Métodos - Dentre os 5870 prontuários de pacientes com HIV em acompanhamento no Serviço de Infectologia do Hospital Nossa Senhora da Conceição, Porto Alegre, RS, foram aleatoriamente selecionados 587 prontuários. Destes, foram considerados para análise retrospectiva aqueles em que havia sido pesquisado algum marcador para hepatites B (HBsAg, anti-HBc ou anti-HBs) ou C (anti-VHC), perfazendo o total de 343 pacientes. Resultados - O HBsAg foi positivo em 14 de 306 (4,6\%) pacientes, anticorpo anti-HBs, em 40 de 154 (26,0\%) pacientes, e anti-HBc em 79 de 205 (38,5\%) pacientes. Dentre aqueles que realizaram anti-VHC, 126 de 330 (38,2\%) tinhamno positivo. Co-infecção por vírus B e C foi observada em 7 dentre os 296 pacientes que realizaram tanto o HBsAg, quanto o anti-VHC (2,4\%). Dentre aqueles HBsAg positivos, a principal categoria de exposição foi o relacionamento homossexual masculino (50,0\%). Dentre aqueles anti-VHC positivos, a principal categoria de exposição foi o uso de drogas ilícitas injetáveis $(75,3 \%)$. Naqueles monoinfectados com HIV (185 pacientes), o fator de risco mais prevalente foi relacionamento heterossexual promíscuo ou com cônjuge infectado por HIV em 83 pacientes (44,9\%). Conclusão - Em nosso meio, as coinfecções VHB-HIV e VHC-HIV são freqüentes, sendo observado maior impacto na associação do VHC com o HIV.

DESCRITORES - Hepatite B. Hepatite C. Infecções por HIV. Prevalência. Ambulatório hospitalar.

\section{INTRODUÇÃO}

Atualmente, aproximadamente 33 milhões de pessoas estão contaminadas pelo vírus da imunodeficiência humana (HIV) no mundo, sendo mais da metade adultos residentes na África ${ }^{(33)}$.

A introdução de novos agentes na terapia anti-retroviral (ARV) aumentou a expectativa de vida entre os infectados pelo HIV, permitindo a observação de um número maior de pacientes com cirrose e com suas complicações em casos de co-infecção pelo vírus da hepatite $\mathrm{B}$ (VHB) e C (VHC), bem como a hepatotoxicidade associada ao uso desses medicamentos ${ }^{(39)}$. Estudos recentes realizados na Itália e na Espanha demonstraram que, em infectados pelo HIV, as hepatopatias crônicas são responsáveis por 35\% e $45 \%$ dos casos de óbito, respectivamente ${ }^{(21,25)}$.

O VHB, o VHC e o HIV utilizam as mesmas rotas de transmissão $0^{(1,2,5,35)}$. No entanto, a transmissão parenteral é mais observada com o VHC do que com o HIV, e a via sexual é mais eficiente para a transmissão do $\operatorname{HIV}^{(38)}$.

A prevalência de $\mathrm{VHB}$ e VHC em pacientes infectados pelo HIV varia de acordo com os fatores de risco para a aquisição de VHB, VHC e HIV ${ }^{(39)}$ e com a sensibilidade/ especificidade dos métodos diagnósticos utilizados para identificar a infecção( ${ }^{(27)}$.

Segundo POLES e DIETERICH ${ }^{(24)}$, metade de todos os pacientes infectados pelo HIV são co-infectados com o VHB ou com o VHC, o que causa impacto na qualidade de vida, na sobrevida e nos custos com tais pacientes.

$\mathrm{O}$ presente estudo teve como objetivos determinar a prevalência de marcadores para hepatites $\mathrm{B}$ e $\mathrm{C}$ em uma população de pacientes portadores do HIV, bem como os fatores de risco envolvidos.

${ }^{1}$ Hospital Nossa Senhora da Conceição; ${ }^{2}$ Fundação Faculdade Federal de Ciências Médicas de Porto Alegre, Porto Alegre, RS.

Endereço para correspondência: Dra. Cristiane Valle Tovo - Rua Cel. Aurélio Bitencourt, 115 - apt. 201 -90430-080 - Porto Alegre, RS. E-mail: cris.tovo@terra.com.br 
Tovo CV, Santos DE, Mattos AZ, Almeida PRL, Mattos AA, Santos BR. Prevalência ambulatorial em um hospital geral de marcadores para hepatites B e C em pacientes com infecção pelo vírus da imunodeficiência humana

\section{PACIENTES E MÉTODOS}

Foram revisados de forma retrospectiva os prontuários de pacientes portadores de HIV do Serviço de Infectologia do Hospital Nossa Senhora da Conceição (HNSC), hospital público de nível assistencial terciário, prestador de atendimento à população de baixa renda $\mathrm{e}$ centro de referência para pacientes com síndrome da imunodeficiência adquirida (AIDS), localizado em Porto Alegre, RS.

Os prontuários são arquivados por ordem alfabética e, dentre os 5870 prontuários existentes, foram revisados aleatoriamente 587. Dentre estes, foram considerados para análise os $343 \mathrm{em}$ que havia sido pesquisada a presença de algum marcador para hepatite B (HBsAg, anti-HBc ou anti-HBs) ou C (anti-VHC).

Os anticorpos anti-VHC foram detectados por meio do teste ELISA III, de acordo com as instruções de fabricação (Abbott Axsym System, N.Chicago, IL, EUA).

A presença de $\mathrm{HBsAg}$, anti-HBc e anti-HBs foi verificada por meio de testes comerciais de radioimunoensaio, de acordo com as instruções do fabricante (Abbott Axsym System, N.Chicago, IL, EUA).

Anticorpos para o HIV-1/HIV-2 foram detectados pelo teste ELISA II e "Immune Chromatrographic Assay Determine" (Abbott Axsym System, N.Chicago, IL, EUA). Amostras positivas eram submetidas a confirmação pelo teste de imunofluorescência (Fundação Oswaldo Cruz, Rio de Janeiro, RJ). Resultados indeterminados eram confirmados pelo teste de "western-blot"(14).

Fatores epidemiológicos como o uso de drogas injetáveis (UDI), transfusão sangüínea antes de 1992 e homens que praticam sexo com homens (HSH), bem como outros fatores, tais como exposição ocupacional, cirurgias prévias de grande porte, procedimentos dentários, acupuntura, "piercing", brincos, tatuagens, história de hepatite no passado, contato com pessoas com hepatite, uso compartilhado de escovas de dentes, navalhas ou lâminas, aspiração intranasal de cocaína, história sexual incluindo relacionamento heterossexual promíscuo ou com cônjuge sabidamente infectado, hemodiálise no presente ou no passado e história de transplante de órgãos, foram avaliados.

A pesquisa foi aprovada pelo Comitê de Ética do HNSC.

$\mathrm{Na}$ análise estatística, o programa MsExcel 2000 foi utilizado para tabulação dos dados; o pacote estatístico SPSS 8.0 ("Statistical Package for Social Science") foi utilizado para análise posterior dos resultados. As variáveis qualitativas foram apresentadas em forma de freqüência e percentual. O nível de significância assumido foi de $5 \%$.

\section{RESULTADOS}

A média de idade foi de 34,4 $\pm 10,6$ anos, 206 (62,4\%) eram homens e $254(76,9 \%)$ eram de raça branca.

O HBsAg foi positivo em 14 de 306 (4,6\%). Dentre aqueles em que se pesquisou a presença do anti-VHC, 126 de $330(38,2 \%)$ tinham-no positivo. Co-infecção por vírus $\mathrm{B}$ e $\mathrm{C}$ foi observada em 7 dentre os 296 pacientes que realizaram tanto o $\mathrm{HBsAg}$, quanto o anti-VHC (2,4\%) (Tabela 1$)$.

$\mathrm{O}$ anticorpo anti-HBs foi positivo em 40 de 154 (26,0\%) e o anti-HBc em 79/205 (38,5\%).

Dentre aqueles HBsAg positivos, a principal categoria de exposição foi HSH (50,0\%). Dentre aqueles anti-VHC positivos,
TABELA 1 - HBsAg e anti-VHC em pacientes portadores de HIV

\begin{tabular}{lcccc}
\hline & Anti-VHC neg & Anti-VHC pos & NR & Total \\
\hline HBsAg neg & 185 & 98 & 09 & 292 \\
HBsAg pos & 06 & 07 & 01 & 14 \\
NR & 13 & 21 & 03 & 37 \\
Total & 204 & 126 & 13 & 343 \\
\hline neg = negativo & & & & \\
pos = positivo \\
NR = não realizado
\end{tabular}

a principal categoria de exposição foi o UDI (75,3\%). Outrossim, este mesmo fator de risco foi ainda mais prevalente quando havia associação entre HIV/VHB/VHC (85,7\%). Por outro lado, naqueles monoinfectados com HIV (185 pacientes), o fator de risco mais prevalente foi relacionamento heterossexual promíscuo ou com cônjuge infectado por HIV, tendo sido verificado em 83 pacientes $(44,9 \%)$.

\section{DISCUSSÃO}

Originária de chimpanzés da África Central, a AIDS atingiu proporções epidêmicas nos anos 80 nos Estados Unidos da América e na Europa, onde a maioria das infecções era adquirida através de contato homossexual. Subseqüentemente, houve a disseminação epidêmica para populações heterossexuais ${ }^{(15)}$. Atualmente, a infecção pode ser adquirida através de transmissão heterossexual em $75 \%$ a $85 \%$ dos casos e a transmissão vertical tem emergido como problema de saúde pública em países em desenvolvimento $^{(15)}$, embora nem todos os autores concordem com a magnitude atribuída a esta forma de transmissão ${ }^{(16)}$.

Coincidindo com essas mudanças na epidemiologia da infecção pelo HIV, novas contribuições surgiram no tratamento da doença, destacando-se o papel dos ARV na sobrevida desses doentes.

Como o prognóstico da infecção pelo HIV melhorou significativamente com a terapia ARV, as hepatopatias crônicas, inicialmente meras coadjuvantes no espectro da infecção pelo HIV, têm adquirido destaque e parecem destinadas a ser causa importante de morbidade e de mortalidade na população coinfectada por $\mathrm{VHB}$ ou $\mathrm{VHC}^{(34,35)}$.

$\mathrm{O}$ estado permanente de ativação imune observado nos pacientes com VHC pode influenciar de forma deletéria os pacientes com HIV, favorecendo a replicação do HIV e a destruição das células CD4 ${ }^{(38)}$. Além disso, a co-infecção acelera a evolução da hepatopatia relacionada ao $\mathrm{VHC}^{(2,34,35)}$.

A prevalência de co-infecção VHC-HIV em coortes específicas varia de $15 \%$ a $60 \%{ }^{(12)}$. A grande variabilidade depende dos fatores de risco da população estudada e dos métodos para a detecção do VHC. Taxas mais altas têm sido observadas em estudos de coorte europeus ${ }^{(26,37)}$.

Estudos de prevalência demonstram taxas de co-infecção por VHC-HIV em 4\% a 15\% entre $\mathrm{HSH}^{(6,29)}, 60 \%$ a $90 \%$ entre usuários de drogas injetáveis ${ }^{(8,32,36)}$ e aproximadamente $100 \%$ em hemofílicos que receberam concentrados de fatores de coagulação antes da introdução da inativação pelo calor, em $1987^{(7,28)}$.

Nos EUA, estima-se que em torno de 30\% dos 800000 indivíduos infectados pelo HIV estejam co-infectados pelo $\mathrm{VHC}^{(18,30)}$. No estudo de coorte EuroSIDA, que incluiu dados de 
3048 pacientes infectados pelo HIV, 33\% da população era antiVHC-positiva; entre UDI, mais de $75 \%$ eram co-infectados ${ }^{(36)}$. A magnitude do problema é maior na Espanha, onde pelo menos $50 \%$ dos 130000 pacientes infectados por HIV são co-infectados por $\mathrm{VHC}^{(36)}$. Em alguns subgrupos de pacientes HIV-positivos, como UDI, a prevalência de co-infecção chega a $90 \%{ }^{(18,36)}$.

$\mathrm{Na}$ França, estudo de coorte $^{(26)}$ incluindo 272 pacientes infectados pelo HIV, demonstrou prevalência de 36,4\% de co-infecção pelo VHC, atribuída à alta proporção de UDI $(78 \%)$.

Estudo de coorte suíço ${ }^{(13)}$ avaliou 3111 pacientes portadores de HIV, verificando a presença de VHC em 47,2\%, diagnosticado por ELISA III e confirmado por RIBA.

Entretanto, estudo de coorte norte-americano mais recente ${ }^{(31)}$, envolvendo 1687 pacientes infectados pelo HIV, mostrou prevalência geral de $16,1 \%$ da co-infecção por VHC.

No Brasil, estudo realizado na cidade de Campinas, $\mathrm{SP}^{(23)}$ avaliou 232 pacientes portadores de HIV em período de 3 anos, observando prevalência de anti-VHC de 54\%.

Estudo retrospectivo realizado na cidade do Rio de Janeiro, $\mathrm{RJ}^{(9)}$, analisando 202 pacientes com HIV, observou a presença de anti-VHC em $18(8,9 \%)$, sendo que destes, 33,3\% eram UDI, e $22,2 \%$ hemotransfundidos.

No presente estudo, a prevalência de VHC em pacientes portadores de HIV foi de 38,2\%, semelhante à descrita por SHERMAN et al. ${ }^{(30)}$, LAUER e WALKER ${ }^{(18)}$, SORIANO et al ${ }^{(36)}$. no estudo EuroSIDA, QUARANTA et al. ${ }^{(26)}$ e maior que a observada por FERRAZ et al. ${ }^{(9)}$ e SHERMAN et al. ${ }^{(31)}$ em seu estudo mais recente.

Apesar de ambos os vírus serem transmitidos por sangue contaminado, o VHC tem 10 vezes mais risco de ser transmitido em acidente de punção do que o HIV e é adquirido mais facilmente por usuários de drogas injetáveis do que o HIV ${ }^{(17,40)}$. Estima-se que $0,3 \%$ dos indivíduos expostos a agulhas contaminadas com sangue HIV-positivo serão infectados ${ }^{(10)}$, comparados com $2 \%$ a $8 \%$ dos expostos ao $\mathrm{VHC}^{(3,22)}$.

Dentre aqueles anti-VHC positivos, a principal categoria de exposição foi o uso de drogas ilícitas injetáveis $(75,3 \%)$, sendo ainda mais prevalente quando havia associação entre HIV/VHB/VHC (85,7\%).

É possível que os índices aqui observados estejam aquém da real prevalência de co-infecção, já que existem flutuações no status sorológico e mesmo soro-reversão (negativação do anti-VHC), sendo 2,5 vezes mais freqüente entre os co-infectados por $\mathrm{VHC} /$ $\mathrm{HIV}$, quando comparados com os monoinfectados pelo $\mathrm{VHC}^{(4)}$. A este respeito, deve-se observar que os estudos de FERRAZ et al. ${ }^{(9)}$ e PAVAN et al. ${ }^{(23)}$ também não realizaram teste confirmatório.
Quanto ao VHB, é também importante o conhecimento epidemiológico da co-infecção com o HIV, sendo o dano hepático e a posterior depuração viral mediados por imunidade celular. A depleção das células CD4 que ocorre nos pacientes com HIV, modifica a história natural da hepatite por VHB. Nos pacientes com HIV, há grande replicação de VHB, com maior risco de evolução para cirrose e carcinoma hepatocelular ${ }^{(20,38)}$.

Dentre os pacientes infectados pelo HIV por exposição sexual (homo ou heterossexual), por UDI ou por transfusão de sangue ou hemoderivados, 95\% apresentavam evidência de infecção passada causada pelo VHB (anti-HBs ou anti-HBc no soro), e cerca de $10 \%$ a $15 \%$ eram portadores crônicos (HBsAg positivo) ${ }^{(11,19)}$.

No presente estudo, a prevalência de HBsAg (4,6\%) foi semelhante à observada em outros estudos brasileiros ${ }^{(9,23)}$, bem como ocorreu com a alta prevalência de marcadores de infecção passada $(26,0 \%$ anti-HBc positivos e $38,5 \%$ anti-HBs positivos). Assim, no estudo de FERRAZ et al.(9), onde foram avaliados retrospectivamente 211 pacientes portadores de HIV, 5,5\% eram portadores de $\mathrm{HBsAg}, 63,5 \%$ apresentavam evidência de infecção passada, e 10\%, imunidade adquirida por vacina. Anti-HBc isolado foi identificado em $20 \%$.

Em outro estudo brasileiro ${ }^{(23)}$, que avaliou 232 pacientes HIV positivos, o HBsAg foi positivo em 5,3\% dos casos. No entanto, marcadores de infecção passada (anti-HBc e anti-HBs) estavam presentes em $44 \%$ e $35,3 \%$, respectivamente. No grupo que apresentava algum marcador para infecção pelo VHB, 59 $(54,1 \%)$ eram HSH. Foi menor, no entanto, que em estudos publicados na literatura internacional. Assim, estudo espanhol ${ }^{(27)}$ evidenciou HBsAg positivo, entre HIV-positivos, em 58\% dos $\mathrm{HSH}$, em $27 \%$ daqueles que receberam transfusão, em $21 \%$ dos heterossexuais e em $11 \%$ de UDI.

No presente estudo, dentre aqueles pacientes com $\mathrm{HBsAg}$ positivo, a principal categoria de exposição foi $\mathrm{HSH}(50,0 \%)$, como também observado por PAVAN et al. ${ }^{(23)}$ e RODRIGUEZMENDEZ et al. ${ }^{(27)}$.

Ressalta-se que naqueles monoinfectados com HIV (185 pacientes), o fator de risco mais prevalente foi relacionamento heterossexual promíscuo ou com cônjuge infectado por HIV, o que ocorreu em 83 pacientes $(44,9 \%)$.

Conclui-se do presente estudo que, em nosso meio, a coinfecção VHB-HIV e VHC-HIV é elevada, notadamente a VHC-HIV. Estes dados são de fundamental importância, já que indicam obrigatoriedade de se solicitarem marcadores de vírus das hepatites nos pacientes com HIV. Só assim procedendo teremos condições de avaliar adequadamente esta população de pacientes, oferecendo tratamento para aqueles que preencherem os critérios atuais e favorecendo, assim, melhor sobrevida aos mesmos. 
Tovo CV, Santos DE, Mattos AZ, Almeida PRL, Mattos AA, Santos BR. Ambulatorial prevalence of hepatitis B and C markers in patients with human immunodeficiency virus infection in a general hospital. Arq Gastroenterol. 43(2):73-6.

ABSTRACT - Background - Hepatitis B and C viruses and human immunodeficiency virus share the same route of transmission, and the prevalence of HBV and HCV infection in patients infected with HIV is greater than it is in the general population. Aim - To determine the prevalence of hepatitis B and C markers in a population of patients with HIV as well as the risk factors involved. Patients and Methods - From 5,870 registration forms of patients with HIV of an Infectology Unit, 587 were randomly selected. From these, the 343 which had investigated the presence of any hepatitis B (HBsAg, anti-HBc or anti-HBs) or C (anti-HCV) marker were retrospectively analyzed. Results - HBsAg was positive in 14/306 (4.6\%), anti-HBs was positive in 40/154(26.0\%), and anti-HBc in 79/205 (38.5\%). The anti-HCV test was reactive in $126 / 330$ (38.2\%). HBV and HCV co-infection was observed in 7 of the 296 patients who had both HBsAg and anti-HCV tests (2.4\%). For those who were HBsAg positive, the main exposure factor was homosexual intercourse (50.0\%). For those who were anti-HCV reactive, the main risk factor was intravenous drug use (75.3\%). In the HIV mono-infected (185 patients), the most prevalent exposure risk factor was promiscuous heterosexual practices or sexual intercourse with a spouse infected with HIV (83 patients - 44.9\%). Conclusion - In our environment HBV-HIV and HCV-HIV co-infections are frequent, a greater relevance being observed in the association between HCV and HIV.

HEADINGS - Hepatitis B. Hepatitis C. HIV infections. Prevalence. Outpatient clinics, hospital.

\section{REFERÊNCIAS BIBLIOGRÁFICAS}

1. Alter MJ. Epidemiology of hepatitis C in the West. Semin Liver Dis. 1995;15:5-14.

2. Bruno R, Sacchi R, Puoti M, Soriano V, Filice G. HCV chronic hepatitis in patients with HIV: clinical management issues. Am J Gastroenterol. 2002;97:1598-606.

3. Cardo DM, Culver DH, Ciesielski CA, Srivastava PU, Marcus R, Abiteboul D, Heptonstall J, Ippolito G, Lot F, Mckibben PS, Bell DM. A case-control study of HIV seroconversion in health care workers after percutaneous exposure. N Engl Med. 1997;337:1485-90.

4. Chamot E, Hirschel B, Winstsch J, Robert CF, Gabriel V, Deglon JJ, Yerly S. Loss of antibodies against hepatitis $\mathrm{C}$ virus in HIV-seropositive intravenous drug users. AIDS. 1990;4:1275-7.

5. Di Bisceglie AM. Hepatitis C. Lancet. 1998;351:351-5.

6. Esteban JI, Esteban R, Viladomiu L, Lopez-Talavera JC, Gonzalez A, Hernandez JM, Roget M, Vargas V, Genesca J, Buti M. Hepatitis C virus antibodies among risk groups in Spain. Lancet. 1989;2:294-7.

7. Eyster ME, Diamondstone LS, Lien JM, Ehmann WC, Quan S, Goedert JJ. Natural history of hepatitis $\mathrm{C}$ virus infection in multitransfused hemophiliacs: effect of co-infection with human immunodeficiency virus. The Multicenter Hemophilia Cohort Study. J Acquir Immune Defic Syndr. 1993;6:602-10.

8. Fainboim H, Gonzalez J, Fassio E, Martinez A, Ortegui L, Eposto M, Cahn P, Marino R, Landeira G, Suaya G, Gancedo E, Castro R, Brajterman L, Laplume H. Prevalence of hepatitis viruses in an anti-human immunodeficiency virus-positive population from Argentina. A multicentre study. J Viral Hepat. 1999;6:53-7.

9. Ferraz GGS, Menezes JA, Gazineo JL, Passoni LFC, Lessa MPM, Pinto PTA. Prevalência de infecção pelo vírus $\mathrm{B}$ e $\mathrm{C}$ da hepatite em pacientes com infecção pelo vírus da imunodeficiência humana. Rev Med HSE. 2002;36:6-11.

10. Gerberding JL. Incidence and prevalence of human immunodeficiency virus, hepatitis $\mathrm{B}$, hepatitis $\mathrm{C}$ virus and cytomegalovirus among health care personnel at risk for blood exposure: final report from a longitudinal study. J Infect Dis. 1994;170:1410-7.

11. Gordon SC, Reddy KR, Gould EE, McFadden R, O’Brien C, De Medicna M, Jeffers LJ, Schiff ER. The spectrum of liver disease in the acquired immunodeficiency syndrome. J Hepatol 1986;2:475-84

12. Gow PJ, Pillay D, Multimer D. Solid organ transplantation in patients with HIV infection. Transplantation. 2001;72:177-81.

13. Greub G, Ledengerber B, Battegay M, Grob P, Perrin L, Furrer H, Burgisser P, Erb P, Boggian K, Piffaretti JC, Hirschel B, Janin P, Francioli P, Flepp M, Telenti A. Clinical progression, survival, and immune recovery during antiretroviral therapy in patients with HIV-1 and hepatitis C virus co-infection: the Swiss HIV Cohort Study. Lancet. 2000;356:1800-5.

14. Jackson JB, Parsons JS, Nichols LS, Knoble N, Kennedy S, Piwowar EM. Detection of human immunodeficiency virus type 1 (HIV-1) antibody by western blotting and HIV-1 DNA by PCR in patients with AIDS. J Clin Microbiol. 1997;35:1118-21.

15. Janoff EN, Smith PD. Emerging concepts in gastrointestinal aspects of HIV-1 pathogenesis and management. Gastroenterology. 2001;120:607-21.

16. Keiserman DR, Both CT, Mattos AA, Remião J, Alexandre COP, Sherman KE. Intrafamilial transmission of hepatitis $\mathrm{C}$ virus in patients with hepatitis $\mathrm{C}$ and human immunodeficiency virus co-infection. Am J Gastroenterol. 2003;98:878-83.

17. Kiyosawa K, Sodeyama T, Tanaka E, Nakano Y, Furuta S, Nishioka K, Purcell RH, Alter HJ. Hepatitis C in hospital employees with needlestick injuries. Ann Intern Med. 1991;115:367-9.

18. Lauer GM, Walker BD. Hepatitis C virus infection. N Engl J Med. 2001;345:41-52.

29. Lebovics E, Dworkin BM, Heier SK. The hepatobiliary manifestations of human immunodeficiency virus infection. Am J Gastroenterol. 1988;83:1-7.

20. Manegold C. Reactivation of hepatitis B seen in HIV-infected patients after starting HAART. Clin Infect Dis. 2001;32:144-8.

21. Martin-Carbonero L, Soriano V, Valencia ME, López M, González-Lahoz J. Impact of chronic viral hepatitis on hospital admission and mortality in HIV-infected patients. AIDS Res Hum Retroviruses. 2001;17:1467-71.
22. Mitsui T, Iwano K, Masuko K, Yamazaki C, Okamoto H, Tsuda F, Tanaka T, Mishiro S. Hepatitis $\mathrm{C}$ virus infection in medical personnel after needlestick accident. Hepatology. 1992;16:1109-14

23. Pavan MHP, Aoki FH, Monteiro DT, Gonçales NSL, Escanhoela CAF, Gonçales Jr FL. Viral hepatitis in patients infected with human immunodeficiency virus. Braz J Infect Dis. 2003; 7:253-61.

24. Poles MA, Dieterich DT. HIV and hepatitis virus infection. Curr Infect Dis Rep. 2000;2:177-84.

25. Puoti M, Spineti A, Ghezzi A, Donato F, Zaltron S, Putzolu V, Quiros-Roldan G, Zanini B, Casari S, Carosi S. Mortality for liver disease in patients with HIV infection: a cohort study. AIDS Res Hum Retroviruses. 2001;17:146-71.

26. Quaranta JF, Delaney SR, Alleman S, Cassuto JP, Dellamonica P, Allain JP. Prevalence of antibody to hepatitis C virus in HIV-1 infected patients. J Med Virol. 1994;42:29-32.

27. Rodriguez-Mendez ML, Gonzalez-Quintela A, Aguilera A, Carballo E, Barrio E. Association of HCV and HBV markers in Spanish HIV-seropositive patients in relation to risk practices. Hepatogastroenterology. 2003;50:2093-7.

28. Rumi MG, Colombo M, Grigeri A, Mannucci PM. High prevalence of antibody to hepatitis $\mathrm{C}$ virus in multitransfused hemophiliacs with normal transaminases levels. Ann Intern Med. 1990;112:379-80.

29. Sherman KE, Freeman S, Harrison S, Andron L. Prevalence of antibody to hepatitis C virus in patients infected with human immunodeficiency virus. J Infect Dis. 1991;163:414-5.

30. Sherman KE, Roustrer S, Chung R, Rajicic N. Hepatitis C: prevalence in HIV-infected patients across sectional analysis of the US ACTG. Antivir Ther. 2000;5:64-5.

31. Sherman KE, Rouster SD, Chung RT, Rajicic N. Hepatitis C virus prevalence among patients infected with human immunodeficiency virus: a cross-sectional analysis of the US Adult AIDS Clinical Trials Group. Clin Infect Dis. 2002;34:831-7.

32. Sonnergorg A, Abebe A, Strannegard O. Hepatitis $\mathrm{C}$ virus infection in individuals with or without human immunodeficiency virus type 1 infection. Infection. 1990;18:347-51.

33. Soriano V, Garcia-Samaniego, Bravo R, Gonzalez J, Castro A, Castilla J, Martiny-Odriozola P, Colmenero M, Carballo E, Suarez D, Rodriguez-Piners FJ, Moreno A, del Romero J, Pedreira J, Gonzalez-Lahoz J. Interferon alpha for the treatment of chronic hepatitis C in patients infected with human immunodeficiency virus. Hepatitis-HIV Spanish Study Group. Clin Infect Dis. 1996;23:585-91.

34. Soriano V, Rodriguez-Rosado R, Garcia-Samaniego J. Management of chronic hepatitis $\mathrm{C}$ in HIV-infected patients. AIDS. 1999;13:539-46.

35. Soriano V, Garcia-Samaniego J, Rodriguez-Rosado R, Gonzalez J, Pedreira J. Hepatitis C and HIV infection: biological, clinical, and therapeutic implications. J Hepatol. 1999;31(suppl 1):119-23.

36. Soriano V, Kirk O, Antunes F, Ludgren J. The influence of hepatitis $\mathrm{C}$ on the prognosis of HIV: the EuroSIDA Study. In: XIIIth International AIDS Conference [online]; 2000; Durhan. Disponível em: http://www.iac2000.org).

37. Soriano V, Sulkowski M, Bergin C, Hatzadis A, Cacoub P, Katlama C, Cargnel A, Mauss S, Dieterich D, Moreno S, Ferrari C, Poynard T, Rockstroh J. Care of patients with chronic hepatitis C and HIV co-infection: recommendations from the HIV-HCV International Panel. AIDS. 2002;16:813-28.

38. Soriano V, Miró JM, Garcia-Samaniego J, Torre-Cisneros J, Nunez M, del Romero J, Martin-Carbonero L, Castilla J, Iribarren JA, Quereda C, Santin M, Gonzalez J, Arribas JR, Santos I, Hernandez-Quero J, Ortega E, Asensi V, et al. Consensus conference on chronic viral hepatitis and HIV infection: updated Spanish recommendations. J Viral Hepat. 2004;11:2-17.

39. Spengler U, Rockstroh JK. Hepatitis $\mathrm{C}$ in the patient with human immunodeficiency virus infection. J Hepatol. 1998;29:1023-30.

40. Villano AS, Vlahov D, Nelson KE, Lyles CM, Cohn S, Thomas DL. Incidence and risk factors for hepatitis $\mathrm{C}$ among injection drug users in Baltimore, Maryland. J Clin Microbiol. 1997;35:3274-7. 